\title{
One-shot aortic anastomosis with an automatic stapling gun
}

\author{
Go Watanabe, MD, PhD, Hiroshi Ohtake, MD, PhD, Kenji Iino, MD, PhD, and Shigeyuki Tomita, MD, \\ $\mathrm{PhD}$, Kanazawa, Japan
}

Video clip is available online.

We performed aortic arch replacement with a circular staple gun (Intraluminal Stapler System, ILS; Ethicon Endo-Surgery Inc, Somerville, NJ), an automatic anastomosis device used in gastrointestinal surgery. The features of the ILS are adjustable stapler height and controlled compressed tissue thickness, allowing uniform staple formation and providing a leak-free anastomosis and good hemostasis. The circular staple gun has been used in gastrointestinal operations since the 1980s, but its early use was controversial. Now that the device has been standardized, this first report of clinical use in aortic aneurysm surgery is pivotal and likely to change practice in cardiovascular surgery.

\section{CLINICAL SUMMARY}

A 35-year-old man with suspected impending rupture of aortic arch aneurysm was admitted. The patient had undergone aortic value replacement for bicuspid aortic valve 10 years previously. On examination, a huge fusiform thoracic aortic aneurysm $7 \mathrm{~cm}$ in diameter was found to extend from the ascending aorta to the aortic arch. Left ventricular ejection fraction was decreased to $20 \%$. For surgical treatment, aortic root replacement with aortic arch replacement was planned; however, because this was a reoperation in a patient with very low left ventricular function, a method that minimized the durations of circulatory arrest and aortic crossclamping was necessary. Instead of manual anastomosis, we therefore decided to use the ILS for aortic arch replacement. This procedure was approved by the institutional review board of Kanazawa University, and informed consent was obtained from the patient.

From the Department of General and Cardiothoracic Surgery, Kanazawa University Graduate School of Medical Science, Kanazawa, Japan.

Disclosures: Authors have nothing to disclose with regard to commercial support.

Received for publication Sept 23, 2012; revisions received Nov 14, 2012; accepted for publication Dec 10, 2012; available ahead of print Jan 9, 2013.

Address for reprints: Go Watanabe, MD, PhD, Department of General and Cardiothoracic Surgery, Kanazawa University Graduate School of Medical Science, 13-1 Takara-machi, Kanazawa 920-8640, Japan (E-mail: watago6633@gmail.com).

J Thorac Cardiovasc Surg 2013;145:1132-3

$0022-5223 / \$ 36.00$

Copyright (c) 2013 by The American Association for Thoracic Surgery

http://dx.doi.org/10.1016/j.jtcvs.2012.12.029

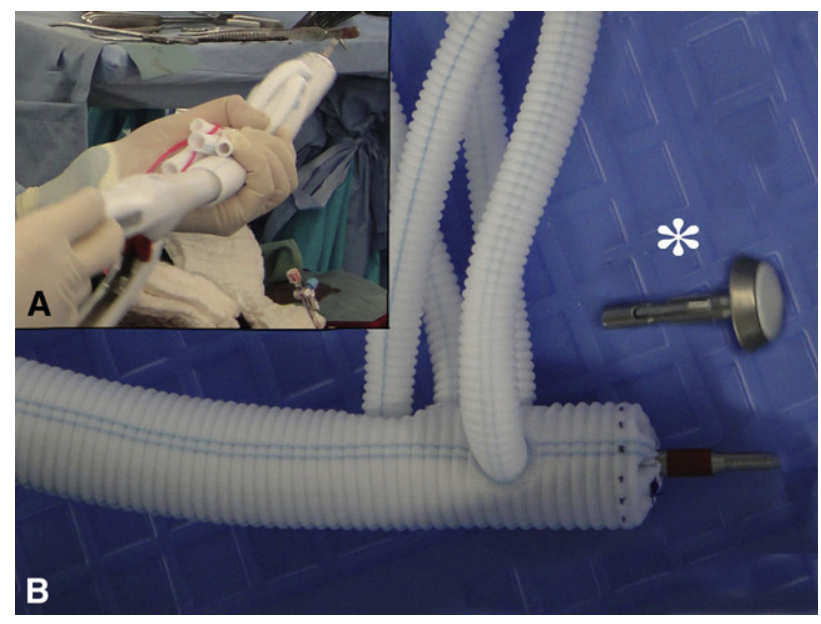

FIGURE 1. A, The circular staple gun preset with a 26-mm 4-branch vessel prosthesis. B, The tip of the staple gun assembly. The end of the vessel prosthesis is gathered in a purse-string fashion. The anvil for insertion into the aortic side is indicated by an asterisk.

Surgery was performed with general anesthesia through a median sternotomy. After cardiopulmonary bypass had been established with 8-mm arterial grafts from bilateral axillary arteries, the adhesions around the heart were removed and the aorta was crossclamped. Selective cerebral perfusion with lower body circulatory arrest was achieved under mild hypothermia at $32^{\circ} \mathrm{C}$. After cardiovascular arrest, the aortic arch was incised, and the descending aorta was transected distal to the 3 branches in the cervical region. To prepare for mechanical anastomosis, a purse-string suture of 3-0 monofilament was placed at the distal stump of the aorta. An anvil was inserted and tied with the purse-string suture. Anastomosis was done with a disposable circular 25-mm stapler preset with a 26-mm 4-branch vessel prosthesis (Figure 1 and Video 1). Anastomosis was confirmed, and the procedure was completed in several seconds. No bleeding from the anastomosis was observed. The subclavian artery, common carotid artery, and brachiocephalic artery were then reconstructed. Aortic root replacement was completed with the Carrel patch technique. The selective cerebral perfusion time was 25 minutes, the circulatory arrest time was 14 minutes, and the cardiac arrest time was 107 minutes. The patient was discharged from the intensive care unit on postoperative day 5 . The postoperative course was uneventful. Computed tomographic scans conducted 3 months after surgery showed no aneurysmal change in the anastomosis (Figure 2). 


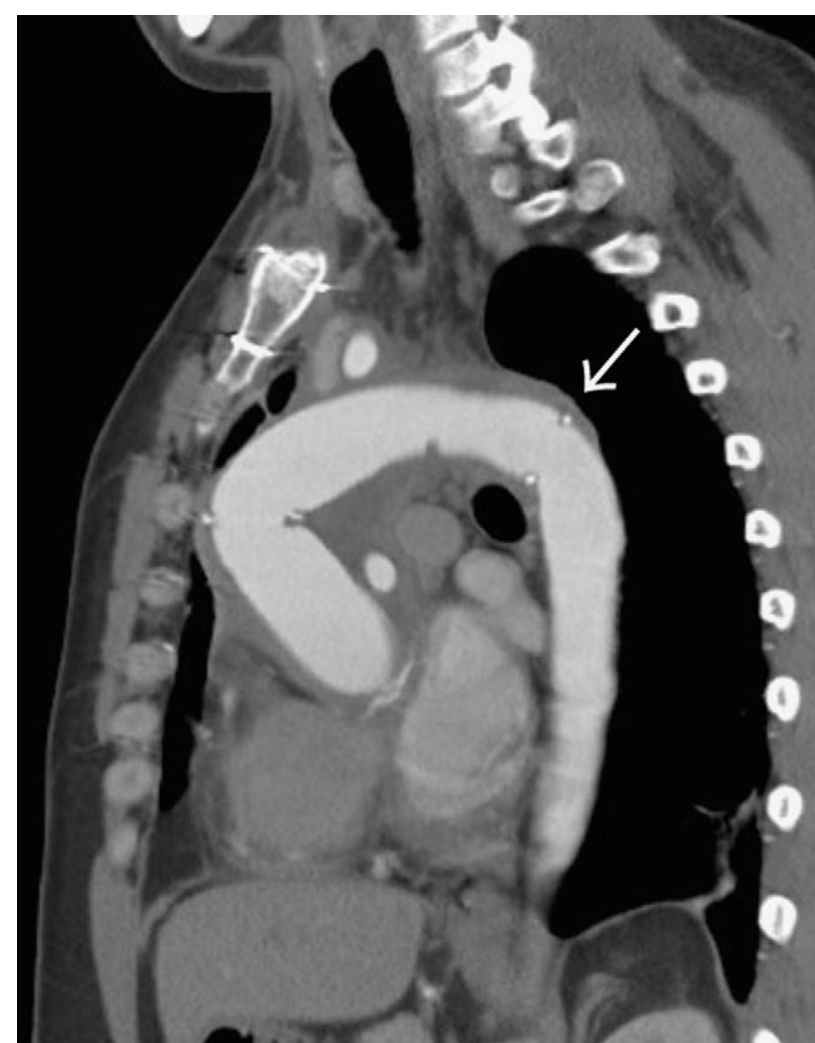

FIGURE 2. Postoperative computed tomographic scan showing good anastomosis and no aneurysm formation or stenosis. The arrow shows the site of a vascular prosthesis anastomosed mechanically by the staple gun.

\section{DISCUSSION}

The use of automatic anastomotic devices has changed the standard of surgery and led to great innovations in surgical technique. ${ }^{1}$ Although automatic anastomotic devices are conventionally used in gastric tube formation and intestinal anastomosis in gastrointestinal procedures, ${ }^{2,3}$ aortic anastomoses are still done by hand suturing. For this reason, operation times are long, and the amount of bleeding depends on the surgeon's skills.
Previously we conducted experimental studies on the use of automatic anastomotic devices for anastomosing the aorta to a vascular prosthesis. ${ }^{4,5}$ The case presented here is the first clinical application of such a device to aortic anastomosis. Use of an automatic anastomotic device in aortic reconstruction for thoracic aortic aneurysm in this patient with low left ventricular function greatly shortened the circulatory and cardiac arrest time and proved to be safe and effective. The ILS has 3 different stapler sizes (21, 25 , and $29 \mathrm{~mm}$ ) that can be used with vascular prostheses ranging from 22 to $30 \mathrm{~mm}$. We believe that vessel sizes from 22 to 30 or even $40 \mathrm{~mm}$ can be anastomosed with the combination. Because both the prosthesis and aorta are inverted when anastomosed, vessels with diameters from $22 \mathrm{~mm}$ (allowing entry of the anvil) to larger than $30 \mathrm{~cm}$ can be anastomosed. Theoretically, the device can be used for the ascending, descending, and abdominal aorta and for the aortic arch. A limitation of the device is that it may not be indicated for acute dissecting aneurysm of the aorta because of the thin wall.

In the future, this method is expected to be useful for patients with poor performance status, cerebral complications, and low left ventricular function. The greatest merit of this method is the short cardiac arrest time, which reduces surgical invasiveness. In addition, the procedure is not technically demanding and can be performed by less experienced cardiovascular surgeons.

\section{References}

1. Göpel T, Härtl F, Schneider A, Buss M, Feussner H. Automation of a suturing device for minimally invasive surgery. Surg Endosc. 2011;25:2100-4.

2. Goligher JC. Use of circular stapling gun with peranal insertion of anorectal pursestring suture for construction of very low colorectal or colo-anal anastomoses. $\mathrm{Br} \mathrm{J}$ Surg. 1979;66:501-4.

3. O'Riordain DS, Buckley DJ, Waldron DJ, Kirwan WO. Purse-string suture for stapled oesophagogastric anastomosis: hand-sewn versus automatic. Br J Surg. 1993; 80:734-6.

4. Takata M, Watanabe G, Ohtake H, Ushijima T, Yamaguchi S, Kikuchi Y, et al. Automatic aortic anastomosis with an innovative computer-controlled circular stapler for surgical treatment of aortic aneurysm. J Thorac Cardiovasc Surg. 2011; 141:1265-9.

5. Yoshida K, Ohtake H, Kimura K, Watanabe G. Experimental study of aortic anastomosis using a circular stapling device in the porcine model. Eur J Vasc Endovasc Surg. 2006;31:575-80. 